\section{Estudo comparativo da concentração salivar do fator de crescimento epidérmico em individuos com laringite crônica por doença do refluxo gastroesofágica antes e após o tratamento: resultados preliminares}

Claudia Alessandra Eckley ${ }^{1,}$ Lilia da Silva Rios ${ }^{2}$, Luiz Vicente Rizzo ${ }^{3}$

\section{Resumo / Summary}

$\mathrm{O}$

mecanismos fisiopatológicos do refluxo laringofaríngeo (RLF) são pouco conhecidos. O Fator de Crescimento Epidérmico (EGF) é a proteína de produção salivar com maior ação na regeneração do epitélio da orofaringe e tubo digestivo alto, tendo sido demonstradas deficiências em sua concentração salivar em indivíduos com RLF. Objetivo: Comparar a concentração salivar de EGF em um mesmo indivíduo com RLF antes e após o tratamento. Material e Método: Neste estudo prospectivo doze indivíduos com DRGE e RLF de moderada intensidade tiveram sua saliva espontânea coletada antes e após o tratamento e controle da doença. A concentração salivar de EGF foi estabelecida através de exame de ELISA (Quantikine ${ }^{\circledR}$ ). Resultados: Onze pacientes eram do sexo feminino e um do sexo masculino, com idade média de 49 anos. A concentração salivar de EGF pré-tratamento foi de $2.867,6 \mathrm{pg} / \mathrm{mL}$ e a pós-tratamento foi $1.588,5 \mathrm{pg} / \mathrm{mL}$, sendo esta diferença estatisticamente significante $(\mathrm{p}=0,015)$. Discussão e Conclusões: Apesar de a concentração salivar de EGF ser maior nos indivíduos antes do tratamento, esta não consegue alcançar àquela de uma população normal (estabelecida previamente), o que sugere uma deficiência primária deste importante fator de defesa em indivíduos com RLF.

\section{Salivary egf concentration in adults with reflux chronic laryngitis before and after treatment: preliminary results}

Palavras-chave: doença do refluxo gastroesofágico (drge), fator de crescimento epidérmico (egf), laringite crônica, saliva Keywords: gastroesophageal reflux disease (gerd), epidermal growth factor (egf), chronic laryngitis, saliva.

\begin{abstract}
$\mathrm{T}$ he Laryngopharyngeal Reflux (LPR) physiopathology is still unknown. The Epidermal Growth Factor (EGF) is a biologically active salivary protein that aids in the rapid regeneration of the oropharyngeal and upper digestive tract mucosas. Salivary deficiency of this protein in patients with LPR has been demonstrated in previous studies. Aim: To compare salivary EGF concentration in patients with LPR before and after treatment. Materials and Methods: In this prospective study twelve patients with GERD and moderate LPR were studied. Whole saliva samples were collected before and after treatment and salivary EGF concentration was determined using a commercially available ELISA kit (Quantikine ${ }^{\circledR}$ ). Results: There were eleven females and one male among the patients, the mean age was 49 years. The mean pre-treatment salivary EGF concentration was 2,867.6 $\mathrm{pg} / \mathrm{mL}$ and the mean post treatment EGF concentration was $1,588.5 \mathrm{pg} / \mathrm{mL}$. This difference was statistically significant $(\mathrm{p}=0.015)$. Discussion and Conclusions: Although salivary EGF concentrations are higher before LPR treatment, the concentration is still much lower than the mean salivary EGF concentration in normal individuals without LPR, which suggests a primary disorder of this defense factor in individuals with LPR.
\end{abstract}




\section{INTRODUÇÃO}

A Doença do Refluxo Gastroesofágico (DRGE) é a doença digestiva mais prevalente da atualidade, sendo implicada em uma gama de alterações do seguimento laringofaríngeo na última década ${ }^{1-17}$. Esta forma supraesofágica da DRGE foi denominada de Refluxo Laringofaríngeo (RLF) por Koufman et al. em 1994 , não com o intuito de designar a origem do refluxo, mas sim de chamar atenção para a predominância de sintomas e alterações no seguimento laringofaríngeo. Sintomas associados ao refluxo são relatados por 3 a $6 \%$ dos indivíduos da população geral semanalmente ${ }^{13,18,19}$. No entanto, pouco se sabe dos mecanismos fisiopatológicos destas manifestações supraesofágicas da DRGE.

É interessante notar como um grande número de pacientes com refluxo laringofaríngeo (RLF), mesmo aqueles com os achados laríngeos mais dramáticos, não tem esofagite ou outros sinais da DRGE no tubo digestivo ${ }^{15-17}$. Certamente, os mecanismos de proteção das mucosas gástrica e esofágica exercem um papel decisivo na capacidade destes órgãos de suportar as agressões mecânicas e químicas a que são submetidos diariamente, sendo vários destes mecanismos mediados pela saliva ${ }^{15,20-27}$. A saliva contém diversas substâncias orgânicas e inorgânicas que contribuem para a proteção contra as agressões físicas e químicas e para a manutenção da integridade da mucosa, não só da cavidade oral como também do tubo digestivo20-26. Os achados clínicos contraditórios e recentes pesquisas sugerem haver deficiências na capacidade de defesa deste seguimento na $\mathrm{DRGE}^{22-24} \mathrm{e}$, mais especificamente, no RLF ${ }^{15}$, 27-29.

Uma das principais responsáveis pela homeostase da mucosa oral e do trato digestivo é a saliva com seu conteúdo orgânico e inorgânico. O Fator de Crescimento Epidérmico (Epidermal Growth Factor - EGF) é a proteína de produção salivar com maior ação na regeneração do epitélio após agressões físicas e químicas por sua grande capacidade de replicar o DNA e a neoangiogênese nas células epiteliais ${ }^{25}$. Em estudo recente comprovamos haver uma diminuição significativa na concentração salivar do EGF em indivíduos com RLF quando comparados a indivíduos normais ${ }^{27}$. O objetivo do atual estudo foi avaliar se ocorrem alterações na concentração salivar de EGF em um mesmo indivíduo com DRGE e RLF antes e após o tratamento clínico a fim de tentar estabelecer se a deficiência deste fator de proteção é primária (congênita) ou secundária (adquirida).

\section{MATERIAL E MÉTODO}

Foram estudados 12 indivíduos, onze do sexo feminino e um do sexo masculino, com média de idade 49 anos (variando de 33 a 72 anos) com diagnóstico clínico endoscópico de DRGE comprovado por uma pH-metria esofágica de dois canais de 24 horas triados de um ambulatório geral de Otorrinolaringologia de um hospital universitário terciário. Este projeto foi aprovado pelo Comitê de ética em Pesquisa Médica da Instituição (número do protocolo CEP 179/04). Somente foram incluídos na pesquisa os pacientes que tiveram diagnóstico endoscópico de DRGE corroborado por pH-metria esofágica de 24 horas de dois canais, consentindo em participar do estudo após esclarecimento sobre os objetivos, procedimentos utilizados e riscos envolvidos. Fatores de exclusão foram tabagismo, etilismo, exposição a químicos inalatórios abrasivos, pois todos estes fatores causam processo inflamatório na mucosa respiratória, podendo mimetizar as alterações encontradas na DRGE. Além disso, também foram excluídos os pacientes que tivessem feito uso de drogas bloqueadoras da secreção gástrica, pró-cinéticos, antiácidos ou anti-inflamatórios hormonais e não hormonais nos 14 dias precedendo a inclusão no protocolo, devido à influência destas drogas na mucosa do aparelho digestivo e na secreção gástrica. Também foram excluídos pacientes com lesões pré-neoplásicas ou neoplásicas da laringe e faringe (presentes ou previamente tratadas). Indivíduos com intolerância às drogas inibidoras de bomba de prótons (IBP) não participaram do estudo.

Todos os participantes responderam a minucioso questionário sobre sua saúde geral e sintomas relacionados à DRGE, suas manifestações digestivas e otorrinolaringoló gicas $^{15,16}$. Também foram submetidos a exame de videolaringoscopia com nasofibroscópio flexível Pentax de $3,5 \mathrm{~mm}$ e tendo seus exames gravados em DVD, usando o escore estabelecido por Belafasky et al. em 2001 (Reflux Finding Score - RFS) (Figura 1) ${ }^{30}$. O exame nasofibrolaringoscópico foi realizado logo no início da participação no protocolo (exame pré-tratamento) e após o término do tratamento e controle da doença (exame pós-tratamento). Consideramos como controle da doença a resolução dos sintomas laringofaríngeos e a melhora do RFS. O tratamento padronizado para todos os pacientes foi droga inibidora de bomba de prótons (IBP) em dose plena via oral antes do café da manhã e antes do jantar por 16 (dezesseis) semanas. Após este período os pacientes foram reavaliados do ponto de vista de sintomas clínicos e sinais laringoscópicos de RLF, sendo nova coleta de saliva feita 7 dias após a interrupção da medicação.

Os participantes do estudo tiveram, assim, duas amostras de saliva total espontânea colhida durante 15 minutos após um período de jejum de 12 horas, uma pré-tratamento e outra pós-tratamento (colhida sete dias após a interrupção do tratamento medicamentoso). Os pacientes foram orientados a não fazerem uso de pasta de dentes no dia da colheita do material, sendo todas as amostras colhidas no mesmo período da manhã para respeitar as flutuações circadianas da produção salivar ${ }^{28}$. As amostras foram imediatamente acondicionadas em 
Figura 1. Escala de intensidade do processo inflamatório causado pelo refluxo laringofaríngeo baseada em sinais videolaringoscópicos (varia de zero a 28).

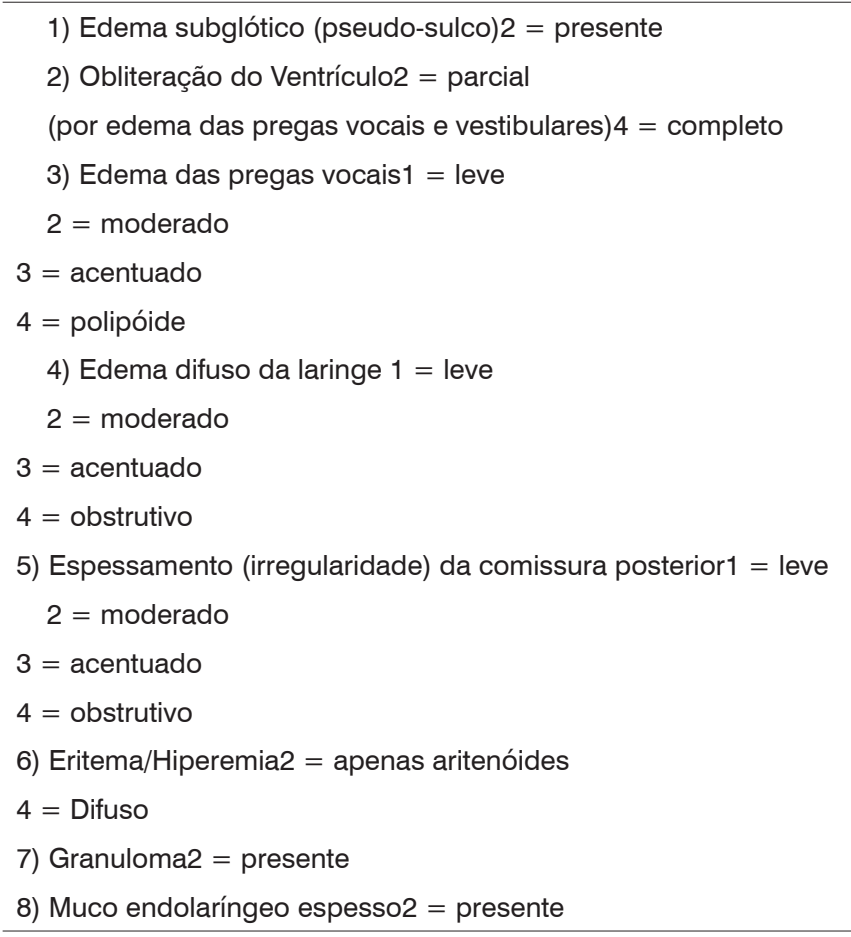

Modificada de Belafsky et al., 2001.

gelo para evitar desnaturação do material protéico a ser estudado. Para o estudo da concentração salivar de EGF foi utilizado o sobrenadante da saliva total após centrifugação da amostra por 10 minutos em temperatura ambiente em uma velocidade de 5000-7000 rotações por minuto (RPM). Este material foi então transferido para outro tubo de Falcon com tampa de rosca e armazenado a 70 graus centígrados negativos até sua análise. As amostras receberam identificação alfa-numérica. Para a determinação da concentração do EGF salivar foi usado um Kit comercial de ELISA para dosagem de EGF (Quantikine R) fornecido pelo R\&D Systems Inc., EUA. A concentração de EGF foi determinada em função da concentração de proteínas da saliva. Foram feitas comparações da concentração salivar do EGF inicial e após tratamento, bem como correlações com melhora da sintomatologia e dos achados inflamatórios laríngeos.

Os resultados foram tabelados e analisados estatisticamente usando-se o teste de Wilcoxon, com nível de significância estabelecido a 95\%.

\section{RESULTADOS}

Todos os participantes do estudo apresentavam RLF em moderada intensidade com sintomas laringofaríngeos diários e um escore médio de sinais (RFS) de 21.4 pontos. Após o tratamento, os pacientes não tinham mais queixas no seguimento laringofaríngeo ou digestivo e o RFS médio abaixou para 13 pontos (Tabela 1). A concentração salivar média de EGF pré-tratamento foi de $2.867,6 \mathrm{pg} / \mathrm{mL}$ e a pós-tratamento e controle da doença foi $1.588,5 \mathrm{pg} / \mathrm{mL}$, sendo esta diferença estatisticamente significante $(\mathrm{p}=0,015)$ (Tabela 1). Comparando a concentração salivar média de EGF da população deste estudo com a concentração salivar de EGF de uma população de adultos normais previamente estabelecida ${ }^{15,27}$, observamos que os indivíduos normais apresentaram em média três vezes mais EGF salivar que

Tabela 1. Dados demográficos e concentração salivar do Fator de Crescimento Epidérmico (EGF) dos indivíduos do estudo antes e após o tratamento com IBP por 16 semanas.

\begin{tabular}{|c|c|c|c|c|c|c|}
\hline pacientes & sexo & idade (anos) & RFS inicial & RFS final & EGF PRÉ (pg/mL) & EGF PÓS (pg/mL) \\
\hline 1 & $\mathrm{~F}$ & 36 & 22 & 13 & 1463,35 & 1071,25 \\
\hline 2 & M & 54 & 21 & 14 & 1874,7 & 56 \\
\hline 3 & $\mathrm{~F}$ & 64 & 22 & 12 & 4717,05 & 2231,15 \\
\hline 4 & $\mathrm{~F}$ & 72 & 20 & 16 & 3006,65 & 2661,3 \\
\hline 5 & $\mathrm{~F}$ & 60 & 23 & 12 & 5337,3 & 365,75 \\
\hline 6 & $\mathrm{~F}$ & 33 & 22 & 14 & 1758,5 & 3316,15 \\
\hline 7 & $\mathrm{~F}$ & 46 & 22 & 12 & 2147,35 & 209,75 \\
\hline 8 & $\mathrm{~F}$ & 45 & 20 & 14 & 3986,3 & 2072,95 \\
\hline 9 & $\mathrm{~F}$ & 38 & 16 & 9 & 2243,65 & 303,7 \\
\hline 10 & $\mathrm{~F}$ & 45 & 24 & 16 & 2633,4 & 2370,35 \\
\hline 11 & $\mathrm{~F}$ & 52 & 24 & 12 & 1729,35 & 1119,2 \\
\hline 12 & $\mathrm{~F}$ & 47 & 21 & 12 & 3516,15 & 3288,2 \\
\hline média & & 49,33 & 21.4 & 13 & 2867,5 & 1588,5 \\
\hline
\end{tabular}


os indivíduos com RLF $(7.085 \mathrm{pg} / \mathrm{mL}$ versus $2.867,6 \mathrm{pg} / \mathrm{mL}$ e $1.588,5 \mathrm{pg} / \mathrm{mL}$ pré e pós-tratamento, respectivamente). Esta diferença entre os indivíduos sem a doença e com RLF foi estatisticamente significante $(\mathrm{p}=0,02)$ (Figura 2). Não houve diferença nos dados quando o único paciente do sexo masculino foi excluído da estatística. Assim sendo, mantivemos os dados deste paciente no grupo estudado.

\section{Comparação da concentração salivar de EGF nos indivíduos com RLF antes e após o tratamento e controle da doença com um grupo controle de indivíduos sem doença}

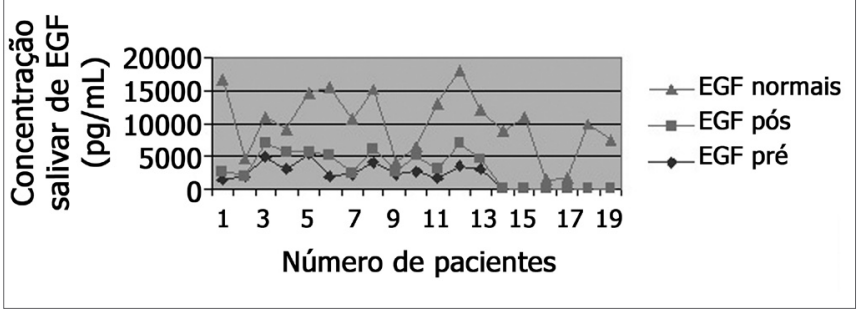

Figura 2. Comparação das médias de concentração salivar de EGF nos dois momentos do estudo (pré e pós-tratamento) com a média de concentração salivar de EGF em população controle sem refluxo.

\section{DISCUSSÃO E CONCLUSÕES}

As formas supraesofágicas da DRGE são de conhecimento relativamente novo para a comunidade científica. Por ocasião das primeiras descrições de quadros de laringofaringite associados a episódios de refluxo havia muita polêmica e incompreensão sobre as formas de diagnóstico e tratamento, já que a maioria das premissas válidas para a forma clássica da DRGE não pareciam se aplicar à forma laringofaríngea. Nesta última década aprendemos a confiar nos achados das endoscopias feitas pelo otorrinolaringologista (videolaringoscopias), não afastando o diagnóstico da doença onde os achados de endoscopia digestiva alta estavam normais ou pouco alterados. Também se aperfeiçoaram as técnicas da pH-metria esofágica, que passou a ter dois canais mensores para melhor avaliar aqueles casos de refluxo fisiológico para o tubo digestivo, mas patológico para a faringe e laringe ${ }^{5,8,11}$. Consensos nacionais ${ }^{31}$ e internacionais ${ }^{32,33}$ foram feitos com o intuito de aproximar a comunicação interdisciplinar e melhor conduzir os casos da DRGE e de RLF. No entanto, ainda não fomos capazes de compreender por completo a fisiopatologia desta doença da sociedade ocidental moderna. Não existe uma correlação entre intensidade de achados endoscópicos, quer no tubo digestivo, quer no seguimento laringofaríngeo, e a intensidade e freqüências dos episódios de refluxo mensurados pela $\mathrm{pH}$-metria de longa duração ${ }^{30,33}$. Indivíduos com a mesma intensidade de episódios de refluxo podem ter alterações inflamatórias da mucosa totalmente distintos. Isto fortalece a possibilidade de haver co-fatores biomoleculares genéticos de pré-disposição para a doença, assim como já determinamos para tantas outras doenças crônicas. Estudos anteriores a este estabeleceram deficiências salivares e no tubo digestivo de fatores de proteção como o EGF em indivíduos com doença dispéptica e esofagite de refluxo ${ }^{21,22,25,26}$. Mais recentemente nós estabelecemos uma significante diminuição na concentração salivar de EGF em indivíduos com laringite crônica por DRGE quando comparados a um grupo de indivíduos normais ${ }^{15,16,27}$.

No atual estudo encontramos uma concentração salivar de EGF significativamente maior nos indivíduos durante a atividade da doença que caiu após o controle da mesma. Esta maior concentração salivar de EGF no período de atividade da doença provavelmente demonstra uma tentativa do organismo em se defender da agressão química à qual está sendo submetido. No entanto, a significante diferença em concentração salivar deste polipeptídeo regenerador do epitélio entre indivíduos normais e com RLF sugere a possibilidade de uma deficiência primária na produção salivar deste importante fator de defesa, o que reforça a maior susceptibilidade de alguns indivíduos para desenvolver alterações inflamatórias na mucosa laringofaríngea após exposição à agressão química oferecida pelo refluxo do conteúdo gastroduodenal.

A DRGE e o RLF sabidamente predominam no sexo feminino ${ }^{3,6,8,15,16}$, o que explica haver apenas um paciente do sexo masculino nesta casuística. No entanto, os dados deste único paciente foram mantidos por estarem homogêneos com aqueles dos demais participantes do sexo feminino. Este estudo relata os resultados preliminares de um estudo maior que visa melhor compreender os fatores fisiopatológicos desta forma atípica (mas tão típica para otorrinolaringologistas) da Doença do Refluxo Gastroesofágico. Se de fato esta deficiência primária nos mecanismos de defesa orgânicos do seguimento laringofaríngeo for comprovada, podemos vislumbrar formas muito menos agressivas e antifisiológicas de diagnosticar e tratar o Refluxo Laringofaríngeo. Apesar da casuística pequena, os achados tiveram grande significância estatística sugerindo uma forte correlação entre deficiência na concentração salivar de EGF e RLF.

\section{REFERÊNCIAS BIBLIOGRÁFICAS}

1. Cherry J \& Margulies SI. Contact ulcer of the larynx. Laryngoscope 1968;78:1937-40.

2. Katz PO. Ambulatory esophageal and hypopharyngeal $\mathrm{pH}$ monitoring in patients with hoarseness. Am J Gastroenterol 1990;85(1):38-40.

3. Koufman JA. The otolaryngologic manifestations of gastroesophageal reflux disease (GERD): a clinical investigation of 225 patients using ambulatory 24-hour $\mathrm{pH}$ monitoring and an experimental investigation of the role of acid and pepsin in the development of laryngeal injury. Laryngoscope 1991;101(Suppl):1-78.

4. Deveney CW, Benner K \& Cohen J. Gastroesophageal reflux and laryngeal disease. Arch Surg 1993;128:1021-6.

5. Paterson WG, Murat BW. Combined Ambulatory Esophageal Ma- 
nometry and Dual-Probe pH-Metry in Evaluation of Patients with Chronic Unexplained Cough. Dig Dis Sci 1994;39(5):1117-25.

6. Fraser AG. Review article: gastro-oesophageal reflux and laryngeal symptoms. Aliment Pharmacol Ther 1994;8:265-72.

7. Koufman JA, Cummins, MM. The prevalence and spectrum of reflux in laryngology:a prospective study of 132 consecutive patients with laryngeal and voice disorders. August 23, 1994. Available Internet $<$ Jkoufman@bgsm.edu and gpostma@bgsm.edu. Center for Voice Disorders homepage $>$ [Jan. 20, 2001]

8. Waring JP, Lacayo L, Hunter J, Katz E, Suwak B. Chronic cough and hoarseness in patients with severe gastroesophageal reflux disease. Diagnosis and response to therapy. Dig Dis Sci 1995;40(5):1093-7.

9. Costa HO, Eckley CA, Fernandes AMF, Destailleur D, Villela PH. Refluxo gastroesofágico: comparação entre os achados laríngeos e digestivos. Rev Port ORL 1997;35(1):21-6.

10. Shaw GY, Searl JP. Laryngeal Manifestations of Gastroesophageal Reflux before and after Treatment with Omeprazole. S Med J 1997;90(11):1115-22.

11. Eckley CA, Marinho V, Ruiz WS, Costa HO. O uso da pH-metria esofágica de dois canais no diagnóstico da laringite crônica por refluxo gastroesofágico. Rev Bras ORL 1999;66(2):110-14.

12. Johanson JF. Epidemiology of esophageal and supraesophageal injuries. Am J Med 2000;108(4A):99S-103S.

13. Cianci $\mathrm{R}$ et al. Is the alkaline reflux a risk factor for laryngeal lesions? Am J Gastroenterol 2000;95(9):2398 (CARTA).

14. Hanson DG, Jiang JJ. Diagnosis and management of chronic laryngitis associated with reflux. Am J Med 2000;108(4A):112S-19S.

15. Eckley CA. Estudo da concentração salivar do fator de crescimento epidérmico em indivíduos com laringite crônica por refluxo laringofaríngeo. São Paulo, 2002. (Tese - Doutorado - Faculdade de Ciências Médicas da Santa Casa de São Paulo).

16. Eckley CA, Costa HO. Estudo da concentração salivar do fator de crescimento epidérmico em indivíduos com laringite crônica por refluxo laringofaríngeo. Rev Bras ORL 2003;69(5):590-7.

17. Gavazzoni FB, De Ataíde AL, Herrero Junior F, Macedo Filho ED Esofagite por refluxo e laringite por refluxo: estágios clínicos diferentes da mesma doença? Rev Bras ORL 2002;68(1):86-90.

18. García-Compéan D, Gonzalez GG, Mar DA, Trevino RM, Bosques F, Maldonado H. Prevalence of gastroesophageal reflux disease in patients with extraesophageal symptoms referred from otolaryngology, allergy, and cardiology practices:a prospective study. Dig Dis 2000;18:178-82
19. Kulig M et al. Quality of life in patients with gastroesophageal reflux disease. Abstracts of the Digestive Disease Week 2002;S1278:A-253

20. Helm JF et al. Acid neutralizing capacity of human saliva. Gastroenterol 1982;83:69-74

21. Helm JF, Dodds WJ, Hogan WJ. Salivary response to esophageal acid in normal subjects and patients with reflux esophagitis. Gastroenterol 1987;93:1393- 7 .

22. Li L et al. Effect of esophageal intraluminal mechanical and chemical stressors on salivary epidermal growth factor in humans. Am J Gastroenterol 1993;88(10):1749-55.

23. Tobey NA. How Does the Esophageal Epithelium Maintain its Integrity? Digestion 1995;56(Suppl. 1):45-50.

24. Sarosiek J, Mccallum RW. Do salivary Organic Components Play a Protective Role in Health and Disease of the Esophageal Mucosa? Digestion 1995b;56(Suppl. 1):32-7.

25. Marcinkiewicz M, Grabowska SZ, Czyzewska E. Role of Epidermal Growth Factor (EGF) in Oesophageal Mucosal Integrity. Curr Med Res Opin 1998;14(3):145-53.

26. Marcinkiewicz M et al. The Potential Role of the Esophageal PreEpithelial Barrier Components in the Maintenance of Integrity of the Esophageal Mucosa in Patients with Endoscopically Negative Gastroesophageal Reflux Disease. Am J Gastroenterol 2000;95(7):1652-60.

27. Eckley CA, Michelsohn N, Tadakoro CE, Rizzo LV, Costa HO. Salivary EGF concentration in adults with reflux laryngitis. Otolaryngol Head \& Neck Surg 2004;131(4):401-6.

28. Dawes C. Circadian rythms in human salivary flow rate and composition. J Physiol 1972;220:529-45.

29. Sonnenberg A et al. Salivary Secretion in Reflux Esophagitis. Gastroenterol 1982;83:889-95.

30. Belfasky PC, Postma GN, Koufman JA. The validity and reliability of the Reflux Finding Score (RFS). Laryngoscope 2001;111(8):1313-7.

31. Moraes-Filho JPP et al. Brazilian consensus on gastroesophageal reflux disease: proposals for assessment, classification, and management. Am J Gastroenterol 2002;97(2):241-8.

32. Koufman JA, Aviv JE, Casiano RR, Shaw GY. Laryngopharyngeal Reflux: Position statement of the Committee on Speech, Voice, and Swallowing Disorders of the American Academy of Otolaryngology, Head and Neck Surgery. Otolaryngol Head Neck Surg 2002;127(1):325.

33. Jaspersen D et al. Prevalence of extra-esophageal manifestations of gastroesophageal reflux disease: an analysis based on the ProGERD Study. Aliment Pharmacol Ther 2003;17:1515-20. 